\title{
SAGE Urban Operations Tool Suite Methodology
}

\author{
John Egan, Daniel Eo, Matthew Hughes, and Jason Lee \\ United States Military Academy, John.Egan, Daniel.Eo, Matthew.Hughes2, and Jason.Lee@usma.edu
}

\begin{abstract}
As the US Army transitions to a regionally aligned architecture, analysts are seeking new and innovative geospatial applications to inform the operations planning process for unfamiliar terrain. In this paper, we outline our systems approach used to develop a suite of geospatial analysis tools used to plan military operations in the urban environment as part of a larger research effort to develop tactical geospatial tools for the US Army (Situational Awareness Geospatially Enabled (SAGE)). This paper describes the iterative stakeholder analysis used to accurately identify operational needs, requirements, capability gaps and associated importance, and the comprehensive methodology used to generate the suite of tools. This research was unique in that we simultaneously developed a recommended suite of tools in addition to a formalized geospatial tool development methodology for the clients' later use. This paper describes the development and application of this methodology which categorically decomposed military operations, aligned stakeholder requirements with existing capabilities, and architected new geospatial capabilities consistent with the existing geospatial program architecture. Key to the developed methodology is that it produced geospatial tools which capitalized upon previously developed technologies, maintained the program's operational theme, and logically developed capabilities which directly satisfied requirements.
\end{abstract}

\section{INTRODUCTION}

\section{Context}

The United States military, despite its strength and capabilities, is not impervious to failures as seen in 1993 when the military experienced operational failures while fighting against urban guerrilla warfighters in the Battle of Mogadishu, Somalia. The U.S. has been involved in urban operations from World War II to the Iraq War and will continue to combat the challenges that urban warfare poses, particularly the effect of urban terrain on warfighters. The utility of an urban operation planning tool has become increasingly relevant and feasible with technological advances in geospatial data and analysis. These lessons learned combined with the future trends of urbanization highlight the necessity for an enhanced and modern aid for urban terrain analysis, which would help warfighters mitigate terrain uncertainties and their consequential dangers in urban operations.

\section{Background}

The Situational Awareness Geospatially Enabled (SAGE) tool suite, developed by Engineer Research and Development Center (ERDC) of the U.S. Army Corps of Engineers (USACE), utilizes geospatial data and specific user parameters to analyze terrain for operations. The objective of our Cadet Capstone Team (CCT) is to incorporate the existing SAGE tools into an urban geospatial planning tool well informed by stakeholder analysis and research. Ultimately, the tool will serve to enhance situational awareness and support mission planning at the tactical, operational, and strategic levels of the U.S. Army. We provide a methodology using a systems engineering approach that will establish a foundational decision framework to aid the creation of new tools.

\section{Problem Definition ANd ReSEARCH}

\section{Research}

In order to build a basic understanding of urban operations and factors in the urban environment, our team explored four research domains. The research domains were History of Urban Operations, Urban Operations Doctrine, GIS Processes Applications, and GIS in Human Geography. We derived these domains from the initial problem which can be divided into two overarching themes, Urban Operations and Geospatial Technology. Furthermore, within the theme of urban operations, it is important to consider US involvement in previous urban operations and current military doctrine to establish a relevant scope. Similarly, we divided geospatial technology as it pertains to two common geographical fields, human and physical. These four domains, we believed, would provide both a wide breadth and depth in understanding the problem.

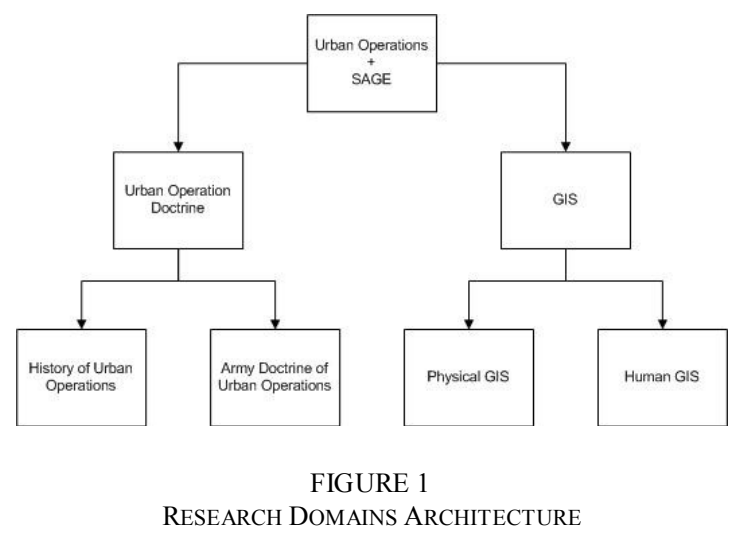

RESEARCH DOMAINS ARCHITECTURE 


\section{History of Urban Operations}

The United States has a rich history of military operations in urban environments leading the US Army Research and Development Center to determine a need for increased planning capabilities in the urban environment. From American involvement in Panama, through the end of the Iraq war, the US Army has fought in urban terrain, including in Desert Storm, Haiti, Somalia, Bosnia, and Kosovo [1] [2. From this growing trend of urban military operations, it is very likely that the US will continue to fight the urban fight in the future, fueling the development of urban planning tools and suites for use in urban operations.

\section{Urban Operations Doctrine}

By investigating the current and future frameworks of urban operations through a doctrinal lens, we can better understand the parameters of the situations that the warfighter experiences in urban theatres and the boundaries in which urban terrain analysis must operate. The goal was to establish the foundational geospatial parameters for the creation of tools that will, at the least, remove the uncertainty of terrain as a disadvantage to the warfighter. Here are the key points of this research thread:

- Army Field Manuals: Military doctrine, specifically FM 3-06: Urban Operations, states that tools should generally aid commanders in understanding the natural terrain around the enemy's defenses, cover and concealed routes, critical objectives such as a vital bridge, and other tactical characteristics of the area and structures. These tools not only give the commander a better overview of the environment, but also allow the commanders to better plan for the operation [3].

- Challenges: The main difficulties in developing a tool for urban environments are due to the complexity of not only the terrain, but also the human geography aspect of urban environments. Consequently, the high density of buildings, population, and structures all pose tactical challenges to the warfighter especially in terms of firing positions, movements, and lines of sight. Additionally, the dimension of collateral damage and noncombatant casualties is often more prominent in urban environments [4].

\section{Physical GIS Applications}

In order to develop a methodology that would combine the technical abilities of SAGE and the military doctrines for urban operations, it was necessary to research the capabilities of ArcGIS, which is used by all geospatial engineers and intelligence analysts across the Army.

ArcGIS is a commonly used program for analyzing various problems associated with physical geographical features. Currently there are many useful SAGE tools which capture doctrinal planning considerations to automate analysis workflow, standardizing the production of tactical decision aides. For example, Braswell discusses several current capabilities to which this urban analysis effort would build upon and improve [5]-[6]. Tools like "Route Analysis" and "Cross Country Movement" identify avenues of approach capabilities for mounted and dismounted troops. These tools operate on elevation data, such as LiDAR (Light Detection and Ranging), land categorization to classify various terrain features as vegetation, ground, or structure and road networks. It is desirable to architect future analysis capability within this structure of the current SAGE effort.

\section{Human GIS Applications}

In examining and mapping the world's urban environment, a stronger focus needs to be directed to a more holistic approach of examining infrastructure - looking beyond the manmade construction to the human residents linked to it. Here are some of the key points identified in this thread:

- Urbanization Mapping: Mapping the people of these cities involve what stage of urbanization they exist in. Coinciding with the culture of the urban environment's population are demographics, such as ranks of poverty and wealth, and religion. Each would have a different impact on conducting urban operations nearby.

- Civilian Considerations and Challenges: The residents of an urban operation introduce complications to situational awareness and threat identification because they inhabit the area of operation and perform different activities. These civilians severely affect movement and maneuver, their culture can affect mission legitimacy, and they often unwillingly help the enemy accomplish their mission [3].

\section{STAKEHOLDER ANAL YSIS}

Our team used two general techniques for stakeholder analysis: interviews and surveys. In order to fully capture the needs of ERDC, and in turn the soldiers in combat, it was important to seek feedback from our clients and users of SAGE technology. The primary populations used for analysis included soldiers of $1^{\text {st }}$ CAV 2-8 Bravo Company, junior officers who have recently commissioned, and company grade and field grade officers who are currently serving at the United States Military Academy. An analysis of these stakeholders provided our team with helpful initial insights for developing and evaluating candidate tools that are valuable to warfighters.

\section{FunCtional AND REQUIREMENTS ANALYSIS}

\section{Functional Hierarchy}

The next step in the Problem Definition Phase is the Functional and Requirements Analysis. These functions are derived from the research and stakeholder analysis conducted in the previous section. The system requirements are specifications that depict the capabilities and levels of performance. Upon the completion of functional and requirements analysis the systems engineer will have a thorough understanding of the functions the solution must perform. 


\section{The Fundamental Objective Architecture}

Our team's first step was to try to break down the various functions of this problem. The initial fundamental objective was to "Support the warfighter's Understanding of the Urban Environment." This objective collectively achieves the goal of ERDC by combining the use of SAGE and the urban environment. When SAGE is expanded to include the urban terrain, it will increase the planning capabilities of the warfighter because the warfighter will be able to better plan and anticipate actions within the urban environment.

Subsequently, our team broke the fundamental objective down into three functions that SAGE would be able to aid the warfighter. These functions were derived to mirror the distinct phases of any Army operation: Planning, Execution, and Assessment.

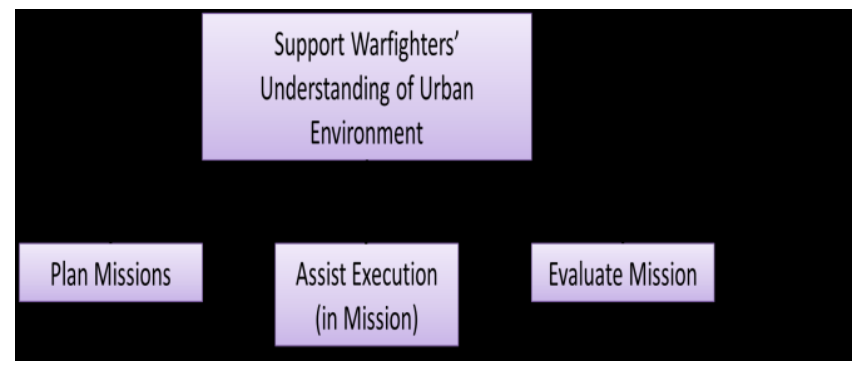

FIGURE 2

INITIAL FUNCTIONAL HIERARCHY

Under the "Plan Missions" function, our group envisioned a SAGE suite of tools that would aid leaders in planning for combat operations in the urban environment. The "Assist Execution" function would be a means to help the warfighter during the execution of the operation and the "Evaluate Mission" function would be a means of conducting an After Action Report. Both of these secondary functions are beyond the scope of effort, thus the fundamental objective became: "Help Warfighter Understand Urban Terrain in Support of Mission Planning."

\section{Mission Planning}

\section{The Urban Environment}

In order to properly address the new fundamental objective of understanding urban mission planning, it was paramount to first define "Urban Terrain". We conducted further research in Army doctrine and determined from FM 3-06: Urban Operations that the urban environment encompassed both physical and human terrain, as well as their interaction [3].

Using this definition, we determined that the urban environment consists of five domains: Natural and Manmade Terrain, which constitutes the "Terrain" or Physical aspect, as well as Civilian Considerations, Enemy Analysis, and Friendly Forces, which encompasses the "Society" or Human aspect of Urban Terrain.

\section{Urban Planning Considerations Decomposition}

Figure 3 portrays the entire decomposition architecture with the two overarching aspects of Physical and Human Terrain located at the diagram's Level 1, down to the five domains located at Level 2, and finally the characteristics of every domain located at Level 3.

- Natural Terrain: This domain encompasses the urban environment's airspace, water, vegetation, elevation, and soil. These features are generally predetermined by nature, and although they can be altered by man, they are not artificial by definition.

- Manmade Terrain: This domain is comprised of the environment's roads and bridges, buildings, utilities, obstacles, and transportation hubs. The infrastructure's physical dimensions, limits, and functions are important to understand in terms of maneuverability and concealment.

- Civilian: When considering Human Terrain, a city's neutral civilian population must be acknowledged, along with enemy units, the warfighter's own unit, and adjacent units occupying the environment. Considerations that involve civilians include demographics, density, location, and their key leaders.

- Enemy/Friendly Forces: For enemy units, the warfighter's unit, and adjacent friendly units, the same components require knowledge and understanding: their demographics, densities, locations, capabilities, influences, and courses of action.

Undoubtedly, these components of Human Terrain are much more complex and abstract, but it is essential to understand both aspects when planning urban operations. Upon completing the entire Urban Mission Planning Decomposition, these aspects were presented, ranked, and analyzed by our population of participating stakeholders in the follow-up "Improved Warfighter Surveys." 


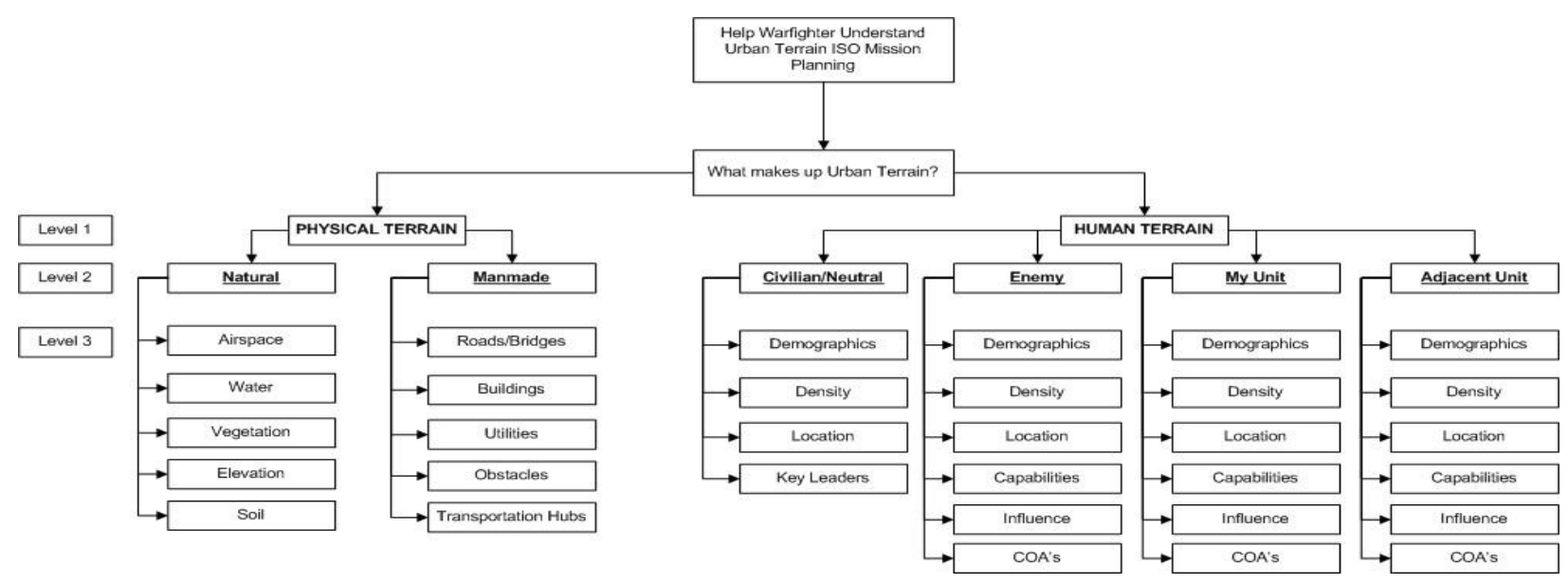

FIGURE 3

URban PLANNING CONSIDERATIONS DECOMPOSITION

\section{REDEFINED STAKEHOLDER ANALYSIS}

The main objective of the redefined stakeholder analysis was to rank the importance of the characteristics within each of the five planning domains. There were three population groups the team assessed: $1^{\text {st }} \mathrm{CAV}$ 1-12 D Company (9 responses), junior officers (14 1LT responses), and 47 USMA cadets (who completed the military science curriculum). The key findings of the results are listed below:

- In the Civilian Consideration domain, our stakeholders believe that understanding civilian leadership (Location of key actors/groups and their influence) is the most important characteristic.

- In the Enemy Situation domain, our stakeholders believe that understanding Enemy Capabilities (Enemy's ability to fight, what the enemy fights with) is the most important characteristic.

- When considering Adjacent Units (Friendly Support), our stakeholders believe that being aware of their location during the mission is most important aspect to know.

- When conducting Man-made terrain analysis (i.e. infrastructure), our stakeholders believe that understanding obstacles (Infrastructure that obstructs communication, maneuvering, and line of sight) is the most important aspect of their mission.

- When conducting Natural terrain analysis, our stakeholders believe that understanding vegetation (location, density, trafficability) is the most important aspect of their mission planning.

\section{Aggregation AND Data AnAlysis}

Figure 4 displays the results of 70 respondents for Survey 2. There are three population groups in this survey: 9 soldiers in a 1st Cavalry Division Armor Company, 14 junior Army officers (Lieutenants), and 47 United States Military Academy cadets. The results in Figure 4 display all the average scores from each population group. The scores are grouped in 5 independent planning domains: Natural,
Manmade, Civilian, Enemy, and Friendly - in that respective order from left to right in the figure below. All five of these domains must be considered when planning an operation in an urban environment. By glancing at Figure 4, we can see the characteristics that are of greatest and least importance to the respondents for each of the domains. For example, in the natural terrain domain, surveyors across the three populations believe location and density of vegetation is the most important characteristic to urban mission planning. Similarly, obstacles that obstruct maneuvering, information regarding civilian leadership, and enemy capabilities received the highest importance in their respective domains across the three survey groups.

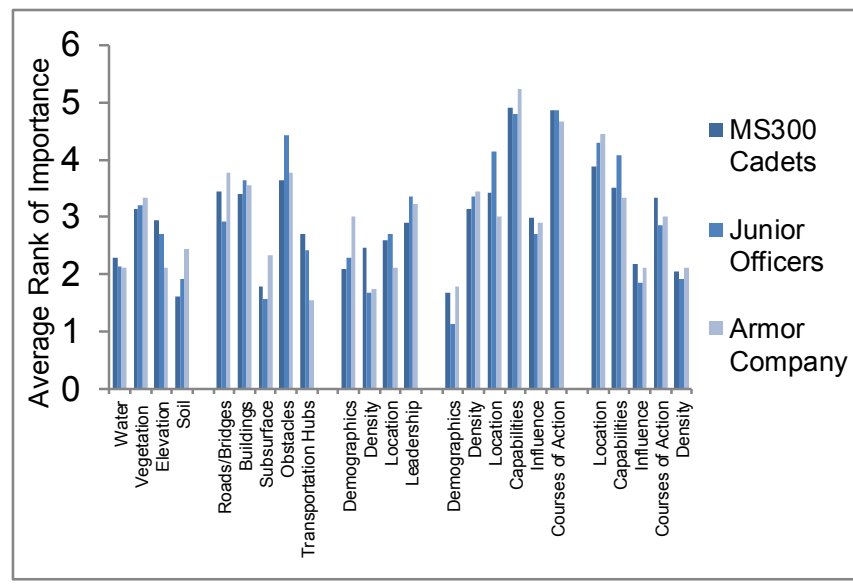

FIGURE 4

SURVEY RESULTS

We then determined the aggregates for each urban characteristic by taking the average of the three responses, weighted by the number of respondents in each of the three populations:

Where $\mathrm{x}=$ Score

$$
A v g=\underbrace{n}_{i=1} \frac{x_{i} * s_{i}}{n}
$$




$$
\begin{array}{ll}
\mathrm{s}= & \text { Population size } \\
\mathrm{i}= & \text { Planning Domain }
\end{array}
$$

Table 1 below displays the normalized results. It is important to reiterate that the results for each of the five planning domains are assessed independently of characteristics in other domains. For example, some considerations in the enemy domain were under-represented because there were 6 considerations that surveyors had to rank whereas some domains can only have a maximum score of 4 . Thus, the characteristics within bigger domains naturally had lower ratings. Nevertheless, we observed that the bottom quarter of the characteristics scored between 0.1 and 0.2 in each domain. Therefore, all urban planning considerations with normalized scores less than 0.2 were not considered for tool concept development.

TABLE I

\begin{tabular}{|c|c|c|c|c|}
\hline \multicolumn{5}{|c|}{ Normalized Scores } \\
\hline & $<0.2$ & $0.20-0.25$ & $0.25-0.30$ & $>0.3$ \\
\hline Natural Terrain & Soil & & Elevation & Vegetation \\
\hline \multirow{2}{*}{ Manmade Terrain } & Subsurface & Roads/Bridges & Obstacles & \\
\hline & Transportation & Buildings & & \\
\hline \multirow{2}{*}{ Civilian } & & Demographics & Location & Leadership \\
\hline & & Density & & \\
\hline \multirow[b]{2}{*}{ Enemy } & Demographics & Density & Capabilities & \\
\hline & Influence & Location & Courses of Action & \\
\hline \multirow{2}{*}{ Friendly } & Influence & Capabilities & Location & \\
\hline & Density & Courses of Action & & \\
\hline
\end{tabular}

SUMMARY OF RESULTS

\section{Alternative Generation Methodology}

In systems engineering, it is necessary to develop a logical means to transition from idea generation to alternative development. Our CCT developed our own methodology to allow for the logical transformation of important planning characteristics found from stakeholder research into SAGE tools that will aid the warfighter. This methodology also incorporates feasibility screening and functional requirement analysis, two important components in the Systems Decision Process. This methodology can be found in figure 5. Upon completion of the initial screening, it is necessary to determine what information is needed with respect to the planning. For instance, for manmade obstacles it is necessary to understand how these obstacles will affect operations. For each point, it is important to determine whether or not a visual or geospatial representation of the information will actually aid in planning. Tools with no visual value will be discarded.

For each point, it is important to determine whether or not a visual or geospatial representation of the information will actually aid in planning. If the information can be displayed in a geospatial form that will be value added for the planner then the information entry will continue through the alternative development methodology. Otherwise, it will be discarded. The next step in the methodology is to decide whether SAGE/ArcGIS already models this information or not. Whether the capability exists, partially exists, or doesn't exist will determine which path it will take through the methodology. The most important part of this methodology is the functional flow diagrams that are created. These diagrams explain, step by step, how each of these tools function. If the diagram shows goodness and value, it will be accepted as an alternative. Else, it will be reformed and then eliminated if it fails to provide the necessary information that is beneficial to the warfighter. All the accepted alternatives continue in the SDP to value modeling.

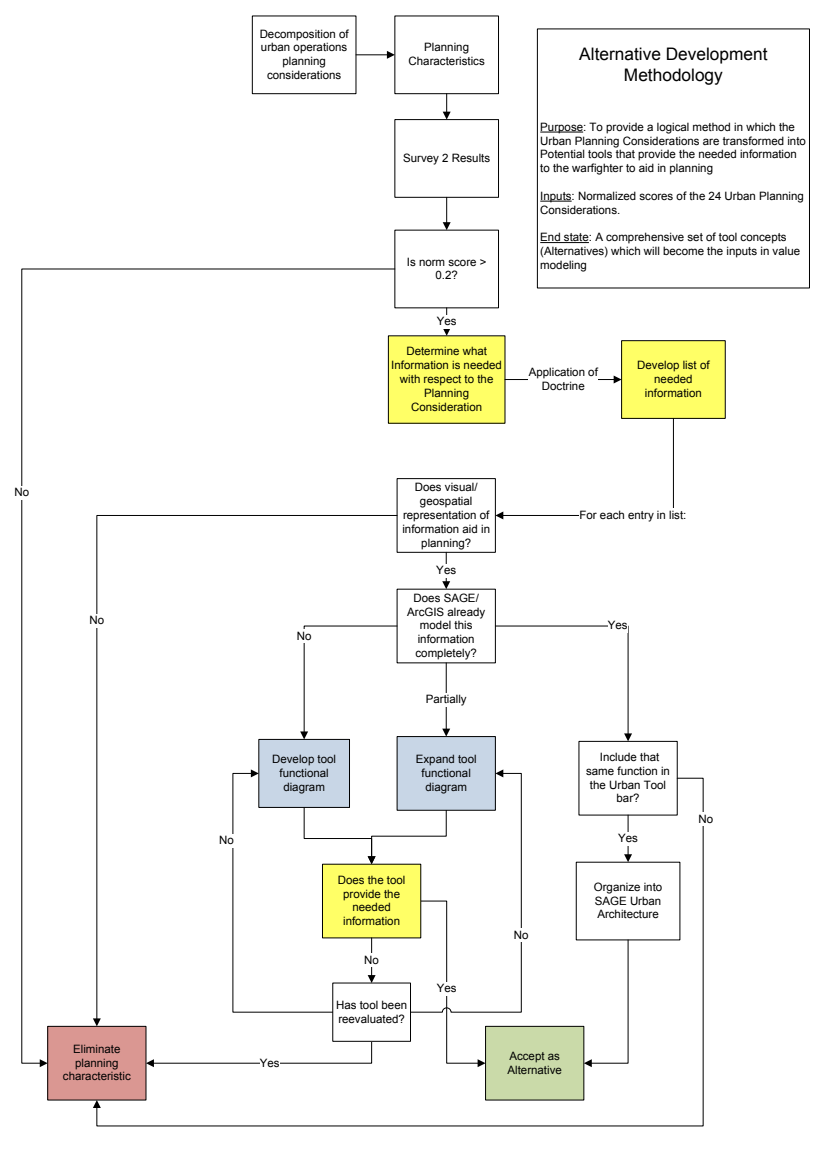

FIGURE 5

ALTERNATIVE GENERATION METHODOLOGY FLOWCHART

\section{FunCTIONAL DiagramMing}

The functional flow diagram is a detailed outline of the systems engineering thought process that goes into the creation of each tool. Using this method, the systems engineer is able to translate the systems decision process to the model builder by combining both doctrinal and technical knowledge of the intended tool. Ultimately, the diagrams act as a foundational framework for the intended functions of the tools and how they can be achieved with current GIS capabilities and data sets. For each alternative, we determined the inputs, outputs, the key processes in between, and the data sets required for each of the following tools. 
- Friendly Forces Location: A tool that can help map and make decisions for locations of Area of Operations (AO) and Quick Reaction Force (QRF) overlays tool which calculates and displays where friendly units are located and to what extent they can perform QRF actions effectively. In order to accomplish these outputs, ArcGIS must be able to either ingest or create an attribute table of friendly forces' locations, types, and boundaries. The next process would involve using the existing Brushfire tool with implementation of SAGE Mobility information. An extract by mask is run on the areas previously defined by the brushfire tool to give overlays of friendly forces QRF coverage, friendly forces QRF non-coverage, as well as adjacent unit QRF coverage.

- Friendly Forces Capabilities: The Friendly Capabilities tool functions to display through overlays the direct and indirect fires capabilities by ingesting data of weapon specifications, location, etc. to create overlays. The intensity of the overlap would show the overlapping reach of the combined friendly forces.

- Enemy Forces Capabilities: The Enemy Capabilities tool is a tool that allows the warfighter to see where the enemies direct and indirect fires capabilities are as well as what the enemy can see based on the visibility conditions. For the enemy tool, it would need the data for the enemy's weapons, both direct and indirect. This tool would be helpful to the warfighter because it would allow a unit to understand where the enemy can hit them. This will affect how the unit will approach the enemy and mitigate as much risk as possible.

- Enemy Location: We did not pursue creating a functional diagram for enemy location because of the lack of data.

- Manmade Obstacles: Our team decided that SAGE could perhaps geospatially represent routes and locations for communication and line of sight. These concepts enable a planning decision in an urban environment under the manmade obstacles domain. To build this tool, we need to either ingest data from various outside sources or create an attribution table of user parameters. The data required for this particular model are elevation and slope data, high fidelity road data, etc. Once the location of obstacles and its dimensions are identified after ingesting the data and plotting them on a map, we can use the routes tools in SAGE to generate both on and off road routes from the air and ground, and locations that obstruct communication.

- Manmade Roads/Bridges: Our team decided that SAGE could geospatially represent maneuvering routes and includes new parameters, road capacity and direction of travel, in order to capture this particular planning consideration. The data required for this concept is traffic density data based on time, high fidelity road data, and direction of travel. Detailed road data to include direction of travel is difficult to obtain because SAGE does not organically use such data. The end-state of this tool concept is to generate optimal on road and off road routes and their respective times of travel.

- Manmade Buildings: The purpose of this tool is to provide the planner with an attribute table of building characteristics such as dimensions and basic calculations in order to save the planner time. The concept of the tool is that the user has the ability to manually mark a boundary of interest where a combination of land classification data, elevation data, and a measuring tool will convert the map graphics to a layer. The tool will be able to automatically calculate basic information such as the number of stories in the building or the width and length of the buildings to provide the planner quick and useful information in urban operations.

- Elevation/vegetation: SAGE already possesses the capability to analyze elevation and vegetation data.

- Civilian: Although our research and stakeholder analysis deemed the tools regarding civilian considerations as highly important, the current SAGE program does not have the proper data sets necessary to perform adequate analysis to aid in planning.

- Density/Demographics/Influence: Lack of data.

\section{CONCLUSION}

Developing a methodology as a framework for the creation of future tools holds great significance with the global increases in urban operations. Further research can be done by evaluating the alternatives with value models and solution design methods. Just as we sought out the needs of the warfighters during our stakeholder analysis, the goodness of the tools will be determined by the warfighters who will ultimately use the tools to aid in the planning of future urban operations.

\section{REFERENCES}

[1] Eversmann, SFC Matthew P. "The Urban Area During Support Missions Case Study Mogadishu." Urban Operations Conference. Ed. Russell W. Glenn. Santa Monica, CA: RAND, 2001. 413-428.

[2] Grange, MG David L. "Training for Urban Operations." 2010. Rand Corporation. Appendix. 30 September 2013.

[3] United States (2006). Urban Operations: Field manual 3-06, Washington, DC: Headquarters, Dept. of the Army.

[4] Glenn, Russell W. Heavy Matter: Urban Operations' Density of Challenges. Rand Corporation, 2000.

[5] Braswell, Kenneth. Route Analysis Toolbar. Alexandria: US Army Geospatial Center, 21 Mar. 2012. MSW.

[6] Braswell, Kenneth. Cross Country Movement (CCM) Models. Alexandria: US Army Geospatial Center, 21 Mar. 2012. MSW.

\section{AUTHOR INFORMATION}

Jack Egan, Daniel Eo, Matt Hughes, Jason Lee, Cadets, Department of Systems Engineering, United States Military Academy. 